\section{Expression of c-Fos Protein in Response to Hypergravity in the Brain of Medaka Fish}

Rie Mizuno, Ryu Miyagawa and Kenichi ljiri*

Radioisotope center, University of Tokyo, Yayoi, Bunkyo-ku, Tokyo 113-0032, Japan

\begin{abstract}
In order to determine the effect of gravity perception on brain activity, we immunohistochemically studied the density of $c$-Fos protein-positive cells in various brain regions of adult wild-type and ha mutant medaka fish. Some ha fish form utricular otoliths (lapilli) in later growing stages and are designated $h a(3-3)$, whereas others continue to lack lapilli and are designated $h a(2-2)$. In most regions, wild-type fish expressed a higher density of c-Fos-positive cells than $h a(3-3)$ and $h a(2-2)$ mutant fish following exposure to hypergravity (3 G) for $3 \mathrm{~h}$. Prominent differences between wild-type and ha mutants were quantitatively demonstrated in the telencephalon, fasciculus longitudinalis medialis, and posterior octavus nucleus with the caudal nucleus. An exception was the stratum periventriculare region, which showed the highest density in ha(2-2) fish and the lowest in wild-types. The results showed that the lapillus is the otolith mainly responsible for the gravity perception of medaka and that the ha mutant can serve as a model for fish born and raised in microgravity. The use of ha fish can help us to understand what changes occur in the gravity-sensing system of medaka fish born and raised for life cycle experiments aboard the International Space Station (ISS). (C2013 Jpn. Soc. Biol. Sci. Space; doi: 10.2187/ bss.27.1
\end{abstract}

Keywords: medaka, otolith, c-Fos, hypergravity, fish, medaka

Received: January 4, 2013; Accepted: January 21, 2013

*To whom correspondence should be addressed:

Tel.: +81-(0)3-5841-2870; Fax: +81-(0)3-5841-3049;

E-mail: ijiri@ric.u-tokyo.ac.jp

\section{Introduction}

The International Space Station (ISS) has now been continuously habited for over 12 years, enabling biologists to carry out long-term space experiments. For those who study fish, the most exciting subject is the realization of fish life cycles in microgravity. In 1994, medaka fish (Oryzias latipes) became the first vertebrates to successfully mate in space. The eggs laid developed normally and hatched in space as fry (baby fish), earning the moniker "space-originated fry" (ljiri, 1995a,b). These fry formed well-developed germ cells within their body, the number of which was the same as the groundhatched fry. Moreover, some of the germ cells in the space-originated fry exhibited signs of starting meiosis (ljiri, 1998), indicating that further generations were also possible in microgravity. Thus, at present, fish are the most likely candidates to be the first vertebrates to complete an entire life cycle in space (ljiri, 2003), and the experiments are due to be carried out soon.

We have been studying the gravity-sensing system of medaka fish for many years (ljiri, 2000; Furukawa and ljiri, 2002; ljiri et al., 2003; Mizuno and ljiri, 2003). In the space station experiments, we are especially interested in what changes occur in the gravity-sensing mechanisms of fish born and raised in microgravity or $0 \mathrm{G}$. Prior to any space experiment, it is always useful to perform groundbased experiments that can help us to understand the implications of the results obtained from the actual space experiments.

The otolithic organ, especially the vertebrate utriculus, is sensitive to the vertical force of gravity. Previously, we used the medaka mutant ha, which lack utricular otolith either bilaterally or unilaterally (ljiri et al., 2003), to study the development and formation of otoliths in both embryos and juvenile fish, finding that four phenotypes with different numbers of otoliths emerge during development (Mizuno and ljiri, 2003). It has also been reported that both efferent and afferent nerve fibers were normally projected, although the shape of the inner ear in the ha mutant showed a somewhat irregular morphology (Noro et al., 2007).

The immediate-early genes, especially $c$-fos, are routinely used for mapping brain activity caused by sensory stimulation in various vertebrate species (Okuyama et al., 2011; Mayer and Bischof, 2012) ever since Hunt et al. (1987) applied the technique to study the effect of physiological stimulation on the activity of the dorsal horn of the spinal cord. In this paper, brain

\title{
Abbreviations
}

BO: bulbus olfactorius, CE: corpus cerebelli, CM: corpus mamillare, CR: crista cerebellaris, DM: nucleus dorsomedialis thalami, E: epiphysis (pineal organ), EC: efferent cells of octavus nerve, flm: fasciculus longitudinalis medialis, G: granule population, $\mathrm{H}$ : hypophysis, LI: lobus inferior, MCN: magnocellular octavus nucleus, MED: medulla oblongata, MN: nucleus medialis, NDLI: nucleus diffusus lobi inferioris, NIV: nucleus nervi trochlearis, nl: nervus olfactorius, NLV: nucleus lateralis, NPPv: nucleus posterioris periventricularis, NRPH: nucleus raphes, nVIII: octaval nerve, OI: oliva inferior, PGc: nucleus preglomerulosus pars medialis commissuralis, PON: posterior octavus nucleus, PON+CAN: PON including the caudal nucleus (CAN), RFm: medial reticular zone, SO: secondary octaval population (medial auditory nucleus of medulla), SPV: stratum periventriculare, TE: telencephalon, TO: tectum opticum, TS: torus semicircularis, $\mathrm{ttb}$ : tractus tectobulbaris, ttbc: tractus tectobulbaris cruciatus, ttbr: tractus tectobulbaris rectus, VC: valvula cerebelli, ved: ventriculus diencephali, vem: ventriculus mesencephali, ver: ventriculus rhombencephali, XL: lobus vagi 

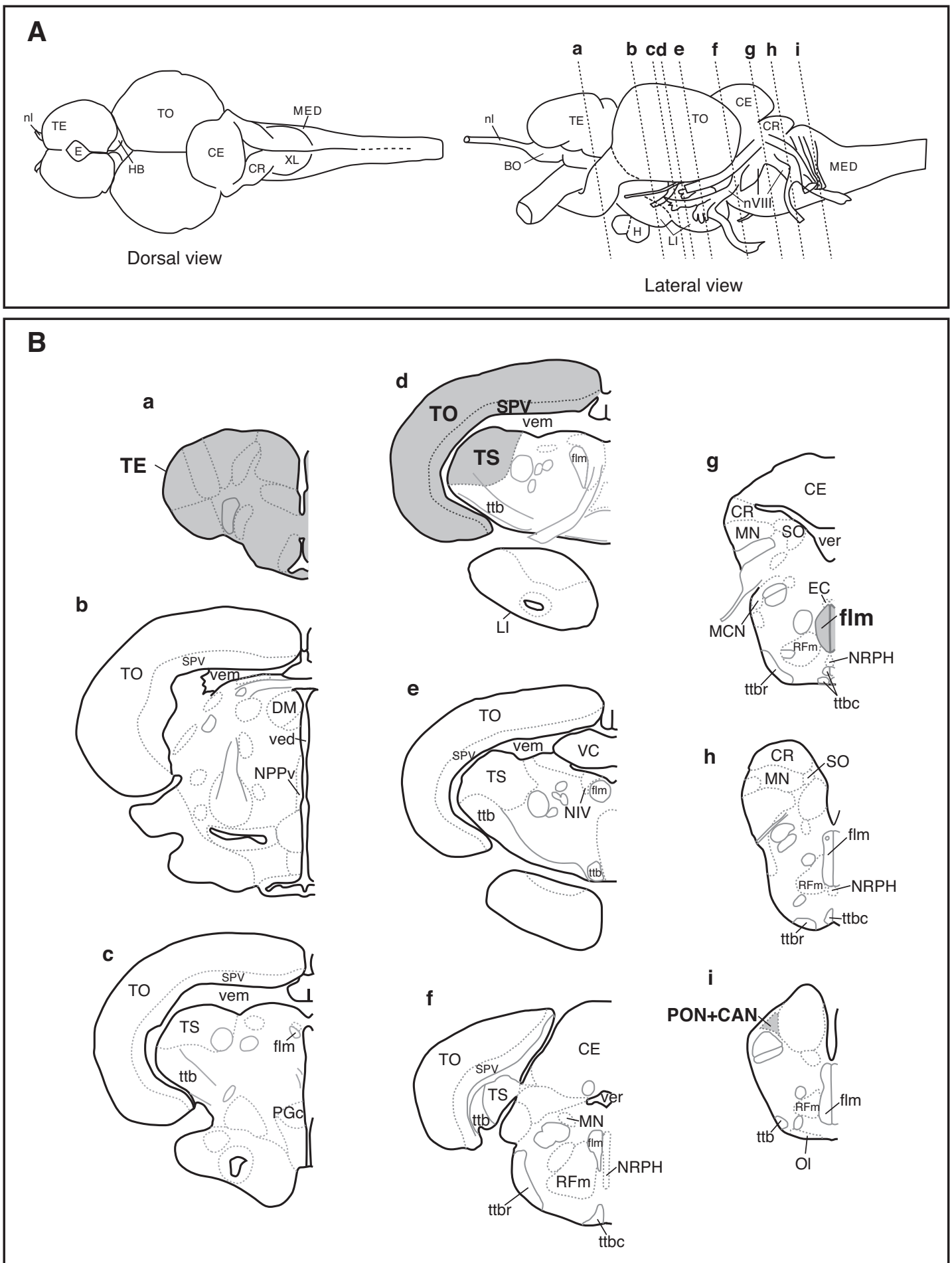

Fig. 1. A: Drawing of the dorsal and lateral views of the medaka brain. Each broken line in the lateral view indicates the cutting level of each section shown in panel B. B: An atlas of the medaka brain. Frontal sections are arranged rostrocaudally from a to $i$. The gray areas are the regions used for counting c-Fos-positive cells to obtain the density data shown in Fig. 5. The meanings of the abbreviations used are listed on the first page of this article. 
activity caused by hypergravity ( 3 G) was examined via immunohistochemical detection of c-Fos protein. Although the brain activity of rats and mice exposed to hypergravity has previously been studied using c-Fos expression (Gustave Dit Duflo et al., 2000; Fuller et al., 2004; Lai et al., 2004), the brains maps of fish and rodents are so different that data for fish are necessary for a full understanding of their hypergravity responses.

At $0 \mathrm{G}$, the utriculus, though physically present in the inner ear, does not have any weight. Thus, it is possible that ground-based studies of the gravity-sensing abilities of mutant ha medaka that lack utricular otoliths can simulate those of normal fish in space $(0 G)$. By comparing the activity of various brain regions in wildtype and otolith-deficient mutant fish (ha), here we tested the possibility that mutant fish can serve as a model fish for studying possible changes in the gravity perception of fish fully grown in gravity-free $(0 G)$ environments.

\section{Materials and Methods}

\section{Animals}

Wild-type and mutant ha medaka (Oryzias latipes) were used throughout this study and their brain responses to hypergravity were examined in various brain regions (Fig. 1; based on the brain map of Ishikawa; see reference). All fish used for the experiments were mature adult fish with a body length $>3 \mathrm{~cm}$. The fish were maintained at a constant water temperature $\left(24^{\circ} \mathrm{C}\right)$ and photoperiod (14 h:10 h light:dark cycle). In order to acclimatize the fish to the environment (e.g., air and light conditions) of the centrifuge room, small plastic vessels, each containing one or two fish with water, were moved to the centrifuge room a few days before the hypergravity experiment was started.

The number of otoliths of the ha mutant fish was determined using a soft X-ray analyzer (EV-70, UNI-HITE SYSTEM Corp., Kanagawa, Japan) in paraformaldehydefixed head samples (Fig. 2). Some ha fish form a utricular otolith (lapillus) bilaterally or unilaterally in later stages of development, while others never develop these lapilli (Mizuno and ljiri, 2003). Thus, two types of ha mutant brain were used in subsequent analyses: those having a full set (3 pairs) of otoliths and designated $h a(3-3)$, and those having only two pairs of lapilli and designated ha(2-2).

\section{Centrifuge-induced hypergravity}

Fully awake adult fish were exposed to 3 $G$ in a centrifuge with two horizontal crossarms (total length: $298 \mathrm{~cm}$ ) at a constant rotation speed (42 rpm, which equals $3 G$ ). Fish in the control group ( $1 \mathrm{G}$ control) were put on the ground of the centrifuge room. Experiments were conducted in normal room light. Fish were exposed continuously to $3 \mathrm{G}$ for $3 \mathrm{~h}$, then decapitated and fixed with $4 \%$ paraformaldehyde in phosphate-buffered saline (PBS). Continuous exposure to $3 G$ was chosen because from our previous study on the appearance of $c$-fos mRNA in this fish (Shimomura-Umemura and ljiri, 2006), it was estimated that the amount of c-Fos protein expressed would show similar values around a $3 G$ exposure time of $2-4 \mathrm{~h}$.

\section{Histological preparation}

Brains were removed and post-fixed in $4 \%$ paraformaldehyde in PBS. After being rinsed in PBS to remove the fixative solution, the brains were dehydrated in a standard graded ethanol series and embedded in paraffin. Frontal serial sections of $14 \mu \mathrm{m}$ thickness were obtained using a microtome.

\section{c-Fos immunohistochemistry}

A search of the medaka c-Fos amino acid sequence (Accession\# NP_001239163.1) in the NCBI protein database was carried out and the most appropriate
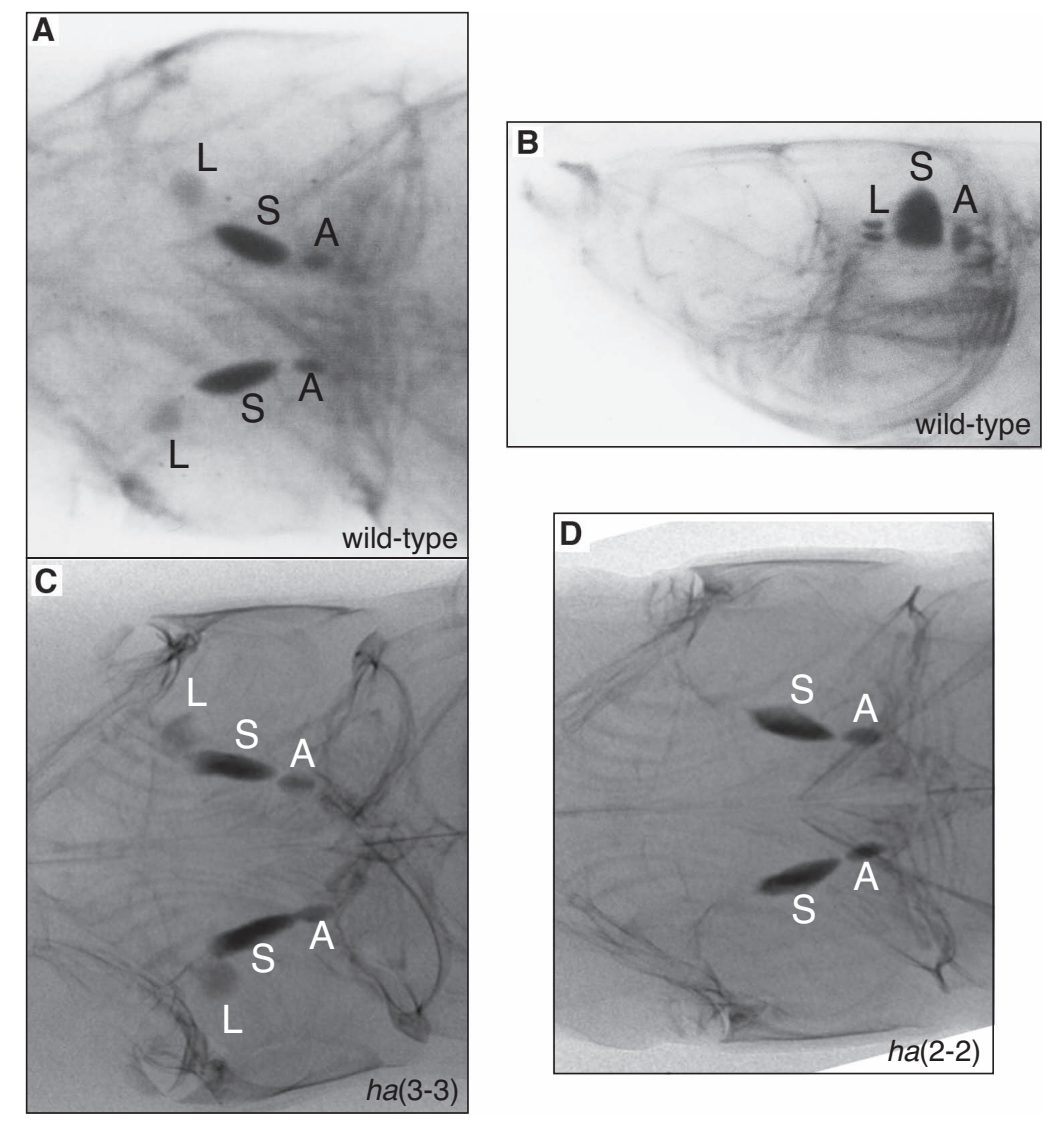

Fig. 2. Soft $X$-ray photographs of the cranium of wild-type (A and $B$ ) and ha mutant $(C$ and $D)$ fish. Two types of ha mutant fish were examined: those, like wild-type fish, with a full set (3 pairs) of otoliths and designated ha(3-3) and those lacking a pair of lapilli and designated as ha(2-2). A: dorsal view of wild-type; B: lateral view of wild-type; C: dorsal view of ha(3-3); D: dorsal view of ha(2-2). All photographs are shown with their rostral tip on the left. The letters in the images indicate the names of the otoliths: L, lapillus; S, sagittal; A, asteriscus. 


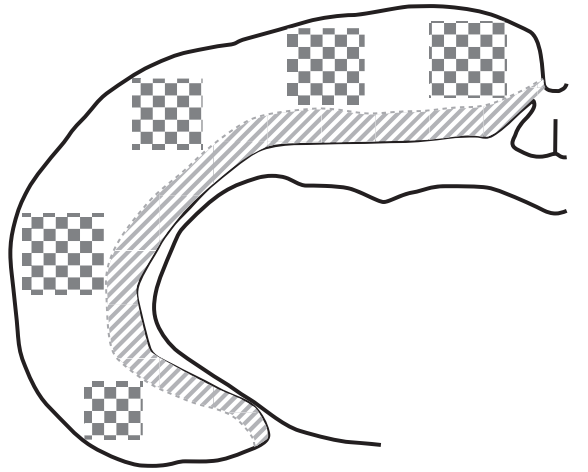

Fig. 3. Schematic illustration of the two different methods employed for calculating the density of c-Fos-positive cells. When the objective area was too large and/or the boundaries were unclear, five or six quadrangle areas were used for measuring the area $\left(\mathrm{mm}^{2}\right)$ and for counting the c-Fos-positive cells within that area (area shown with a checkerboard pattern, e.g., for TE, TO). If the area was small and/or its border was distinct, the whole area was measured and all the c-Fos-positive cells in that area were counted (area with striped pattern, e.g., for SPV, flm, TS, PON+CAN). sequence (residues 214-227; LSTTESSMPTFTSS) for the production of an anti-medaka c-Fos polyclonal antibody was selected.

Tissue sections were deparaffinized with xylene and rehydrated through a graded alcohol series and subsequent rinsing in tap water. In order to quench endogenous peroxidase, sections were incubated with $0.3 \% \mathrm{H}_{2} \mathrm{O}_{2}$ in water for 20 min. Following washing in water and PBS, sections were incubated in blocking solution containing $10 \%$ fetal bovine serum (FBS) in PBS with $0.1 \%$ Triton $\mathrm{X}-100$ for $1 \mathrm{~h}$. Sections were subsequently incubated in anti-medaka c-Fos antibody (1:200; antirabbit polyclonal) in PBS with $10 \%$ normal bovine serum and $0.1 \%$ Triton $\mathrm{X}-100$ at $4^{\circ} \mathrm{C}$ overnight. After primary antibody incubation, the sections were washed five times in PBS containing $0.1 \%$ Triton $\mathrm{X}-100$ (PBST) before being incubated in a 1:1000 biotinylated secondary antibody solution for 1 hour at room temperature. Following five washes, the sections were incubated in avidin-biotin conjugated reagent for $30 \mathrm{~min}$ followed by incubation in 3', 3'-diaminobenzidine (DAB) solution to visualize the c-Fos-positive cells (VECTASTAIN ${ }^{\circledR}$ ABC kit; Vector Laboratories, Burlingame, CA, USA). Finally, sections were rinsed with water and mounted.

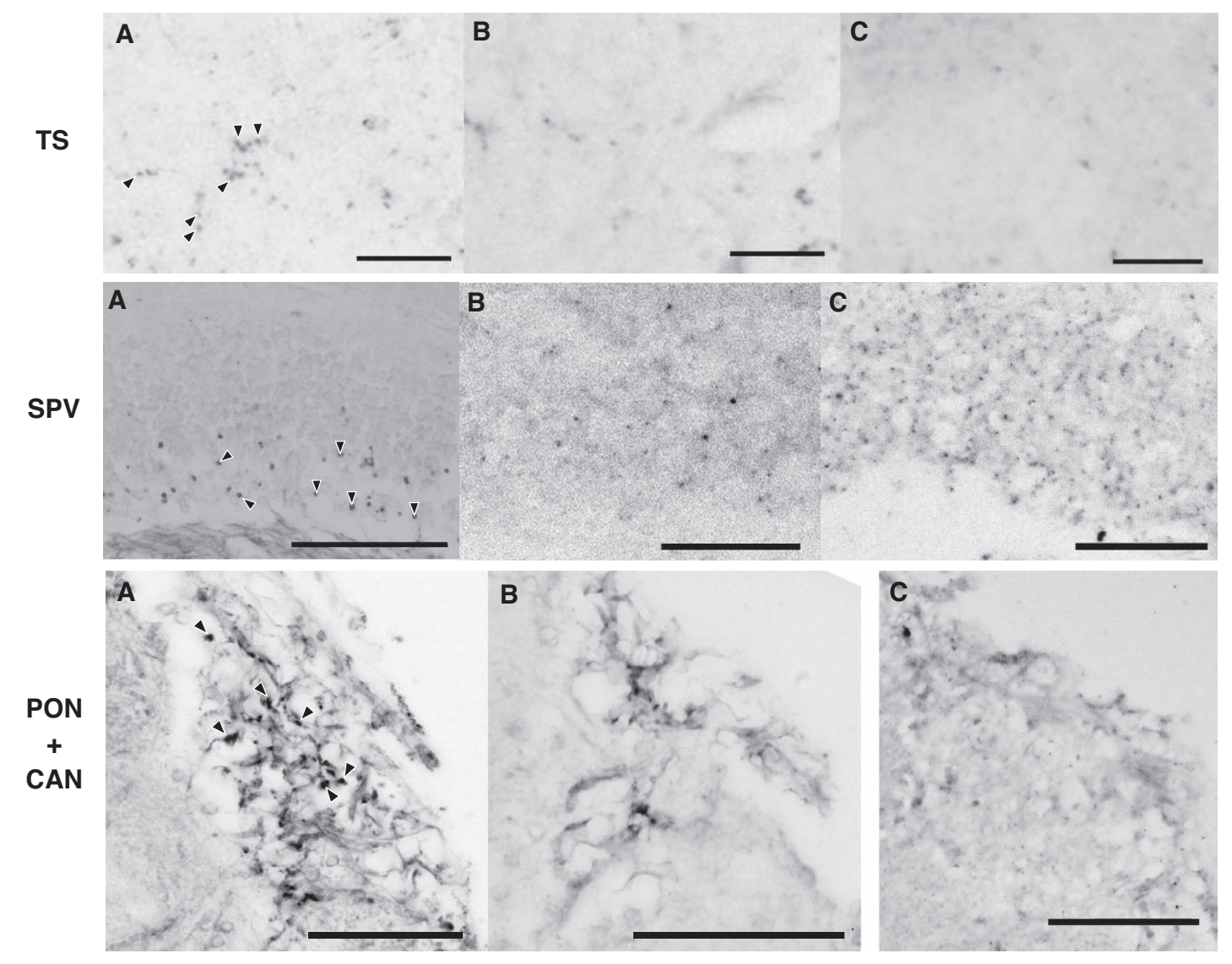

Fig. 4. Photographs of the immunohistochemically stained histological sections. Three regions are shown: top row for TS; middle row for SPV; and bottom row for PON+CAN (for the detailed locations, see Fig. 1). In each row, the region of the right brain in the three types of fish-wild-type (A), ha(3-3) (B) and ha(2-2) (C), respectively-is shown. Several c-Fos-positive cells are indicated with small arrowheads in the wild-type panels. Scale bar for TS represents $20 \mu \mathrm{m}$, whereas that for SPV and PON+CAN represents $50 \mu \mathrm{m}$. 


\section{Cell counting}

In order to count the c-Fos-positive cells, photomicrographs were taken using a digital camera. ImageJ software program (version 1.46; Rasband, 1997) was used to count the c-Fos-positive cells and to measure the total area in which the cells were counted; the c-Fos-positive cell density was obtained from these data. Two different methods were employed for the measurement of the area where c-Fos-positive cells were counted (for details see the explanation of Fig. 3). The brains of five wild-type fish and eight each of ha(3-3) and ha(2-2) were studied.

\section{Results}

Figure 4 shows photomicrographs of the histological sections of the right brain regions of the torus semicircularis (TS), stratum periventriculare (SPV; the sixth layer of the optic tectum), and posterior octavus nucleus including caudal nucleus (PON+CAN) from the three types of fish, namely wild-type, ha(3-3), and ha(22). The ha(3-3) are ha mutant fish that, as adults, have three pairs (a full set) of otoliths. In contrast, ha(2-2) fish have only two pairs of otoliths, even in adulthood. For the TS and PON+CAN regions, more c-Fos-positive cells are seen in wild-type than ha mutants. This is especially prominent in the $\mathrm{PON}+\mathrm{CAN}$ region that has many positive cells, only some of which are marked by arrowheads, in the wild-type (Fig. 4A). However, the SPV region shows the reverse: a large number of positive cells in the ha(2-2) sections (Fig. 4C) and few cells in the wild-type sections (Fig. 4A).

A similar comparison was made under the microscope between the three types of fish for a number of brain regions. The data for all the regions listed in Fig. 1 are summarized in Table 1. Here the appearance patterns of c-Fos-positive cells in the various brain regions are shown in a semi-quantitative way, using four degrees: + + denoting a great number of c-Fos-positive cells; +, a small number of c-Fos-positive cells; -, a few number of the c-Fos-positive cells; and - -, no c-Fospositive cells. In most regions, wild-type fish exhibited a greater density of c-Fos-positive cells than ha(3-3) and ha(2-2) fish. However, as noted in Fig.4, the SPV was an exception: ha(2-2) shows the greatest density and wildtype the lowest.

Six different regions were selected-fasciculus longitudinalis medialis $(\mathrm{flm}), \mathrm{PON}+\mathrm{CAN}$, SPV, telencephalon (TE), tectum opticum (TO), and TS - and the c-Fos cell density in each was calculated from the cell counts per area measured (Fig. 5). For each fish type, both right and left brain density data are shown. The cell density is expressed as cells $/ \mathrm{mm}^{2}$ and note that the ordinate scale for each panel is either in $10^{4}$ cells/ $\mathrm{mm}^{2}$ or in $10^{3}$ cells $/ \mathrm{mm}^{2}$ as the TE and flm of wild-type fish exhibited a higher density, which required that the ordinate scale in their panels be expressed in $10^{4}$ cells/ $\mathrm{mm}^{2}$. The $1 \mathrm{G}$ control fish (kept on the ground in the centrifuge room) and both wild-type and ha mutants showed very few c-Fos-positive cells in any of the brain
Table 1. Distribution of the c-Fos positive cells in the brain of medaka after exposure to hypergravity $(3 G)$ in three types of fish, i.e., wild-type, ha(3-3) and ha(2-2). For abbreviation of each region of brain, see the first page of this paper. The density of c-Fos positive cells was expressed semi-quantitatively in this table, through the microscope examination of each brain region.

\begin{tabular}{|c|c|c|c|}
\hline \multirow{2}{*}{ Area name } & \multicolumn{3}{|c|}{ Type of fish examined } \\
\hline & wild-type & ha (3-3) & ha (2-2) \\
\hline TE & ++ & + & + \\
\hline DM & + & - & - \\
\hline NPPV & + & - & - \\
\hline PGc & + & -- & - \\
\hline TO & + & + & + \\
\hline SPV & - & + & ++ \\
\hline TS & + & + & + \\
\hline ttb & + & - & - \\
\hline NIV & - & - & -- \\
\hline VC & + & + & + \\
\hline flm & ++ & + & + \\
\hline NRPH & + & - & - \\
\hline CE & + & + & + \\
\hline MCN & + & - & - \\
\hline EC & - & - & - \\
\hline CR & - & - & - \\
\hline MN & - & -- & -- \\
\hline NLV & - & -- & -- \\
\hline SO & - & -- & - \\
\hline ttbc & - & - & - \\
\hline ttbr & + & -- & -- \\
\hline $\mathrm{PON}+\mathrm{CAN}$ & + & - & -- \\
\hline OI & + & - & - \\
\hline
\end{tabular}

++ : a great number of the c-Fos positive cells, + small number of the c-Fos positive cells, - : a few number of the c-Fos positive cells, - - : no c-Fos positive cells.

regions examined. For example, in those regions in which total area was measured (i.e., TS, SPV, flm, and PON+CAN), no positive, or at most only a few, cells were encountered in the entire area. Such a low basal expression of c-Fos protein concurs with reports on other animals (Gustave Dit Duflo et al., 2000; Fuller et al., 2004).

Different brain regions contain different numbers of cells of different sizes. Thus, the higher density of c-Fos-positive cells in a certain region does not necessarily mean that the area is especially responsive to hypergravity. Accordingly, the c-Fos-positive cell density data shown in Table 1 and Fig. 5 are not meant as a comparison of the various brain regions but as a comparison of the c-Fos-positive cell density between the three types of fish for each brain region. Thus, as far as each region is concerned, there were no differences in the histological cell arrangement between the brains of the three types of fish. This enables the comparison between the three types of fish with regard to the c-Fospositive cell density observed in each region. 
TE
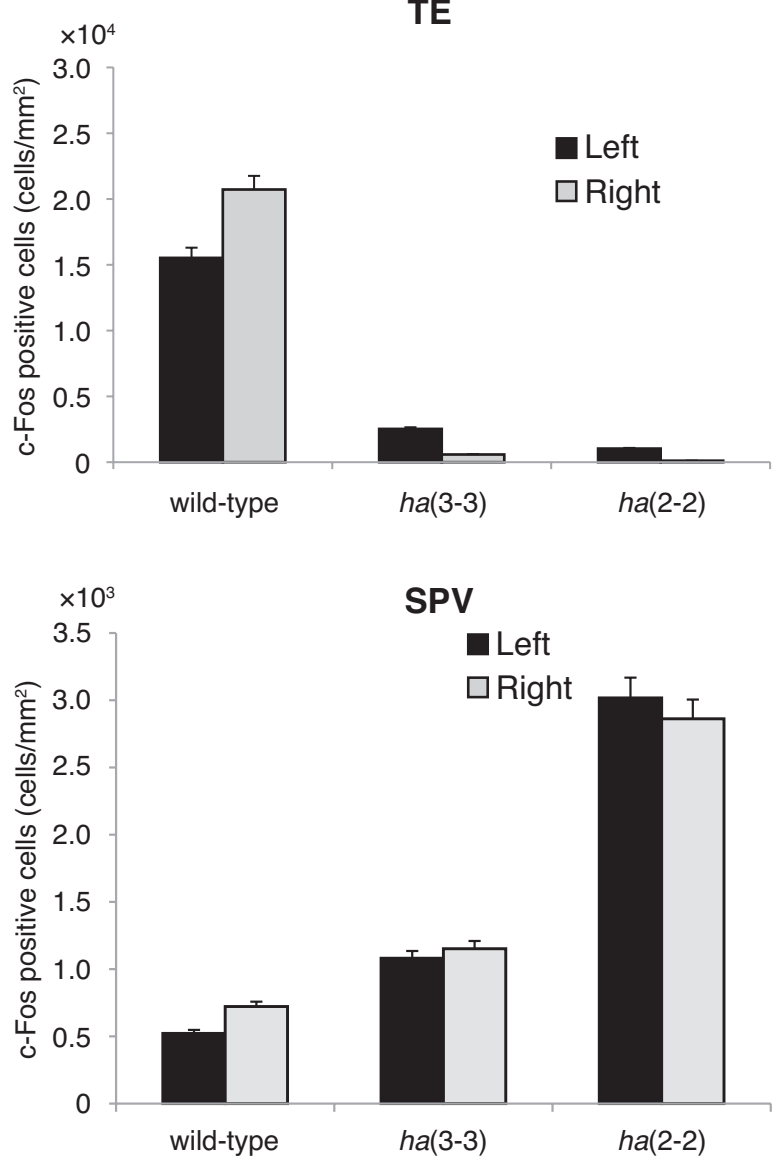

flm

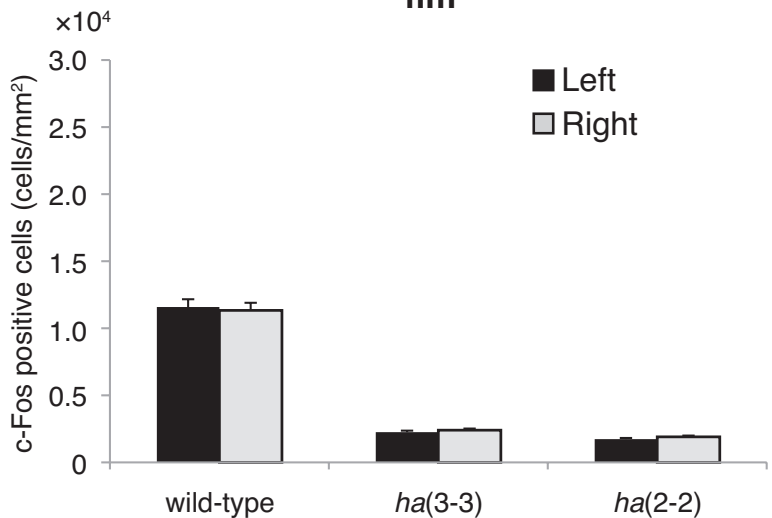

TS

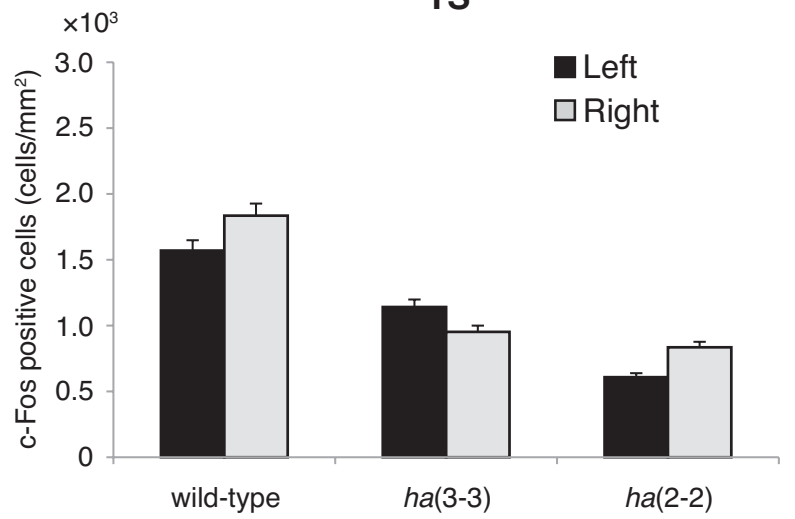

TO

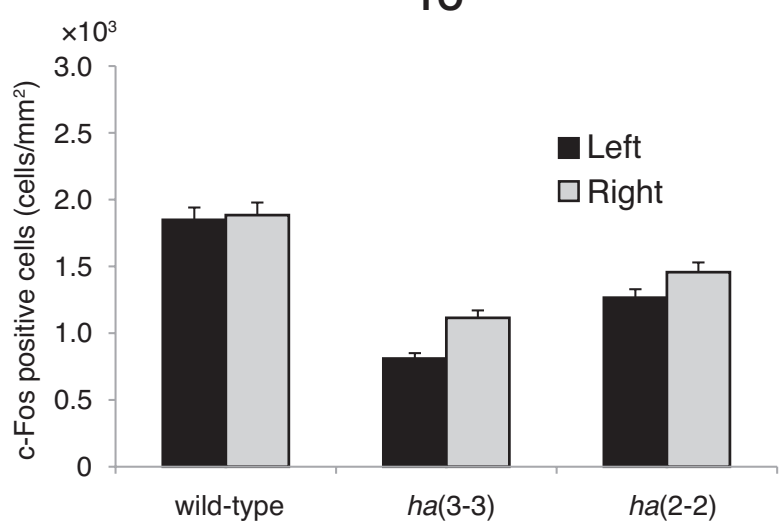

PON+CAN

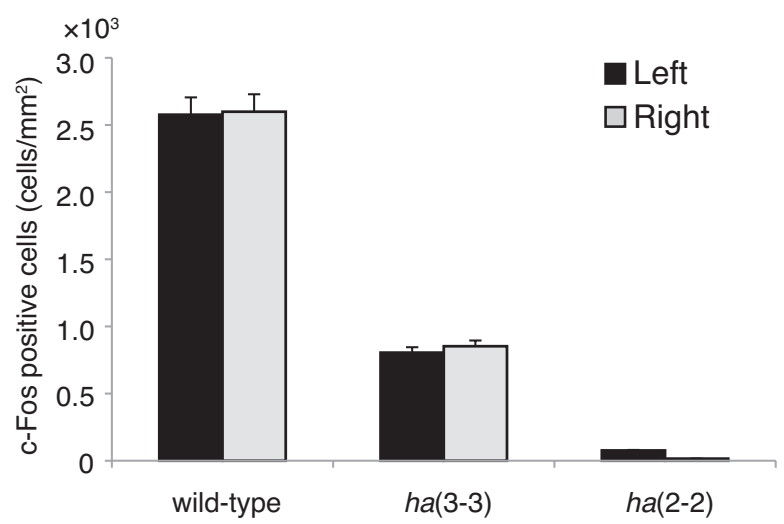

Fig. 5. Density of the c-Fos-positive cells in different regions of the medaka brain after exposure to hypergravity (3 G). Both right and left brains were examined for each type of fish: wild-type, ha(3-3), and ha(2-2). c-Fos-positive cell density was calculated from the area measured $\left(\mathrm{mm}^{2}\right)$ and the number of c-Fos-positive cells found in that area, as detailed in the Materials and Methods. For details of the locations and the full names of the abbreviated regions, see the first page. Bars indicate standard error of the mean. 
A large difference is noted between wild-type and ha (both 3-3 and 2-2) fish in the TE, fIm, and PON+CAN regions. This difference indicates that, although these brain regions of wild-type fish respond actively to hypergravity exposure, the same regions of the ha mutant are less responsive. As expressed semi-quantitatively in Table 1, the SPV of ha(2-2) fish exhibited the highest density, and the lowest density was seen in the wildtype SPV. This is demonstrated quantitatively in Fig. 5. Though not prominent, the TO region shows the highest density in wild-type fish (Fig. 5). The optic tectum has six layers. The five layers from the outermost first to the fifth layers are together designated TO and the sixth layer (the innermost layer) is designated SPV. The present results show the sixth layer (SPV) and the rest of the layers (TO) of the optic tectum respond differently, or even oppositely, to hypergravity exposure.

\section{Discussion}

In most brain regions, though there are slight differences in the density of c-Fos-positive cells between the mutants, both ha(3-3) and ha(2-2) fish show lower cell densities than wild-type fish (see Table 1 and the TE, $\mathrm{flm}$, and $\mathrm{PON}+\mathrm{CAN}$ regions in particular in Fig. 5). As far as the number of otoliths is concerned, both $h a(3-3)$ and wild-type fish have three pairs of otoliths (Fig. 2A and C). However, macroscopically, in the histological sections, the brains of both ha strains always show an irregular morphology different from the normal shape of wild-type brains. The present data for the density of c-Fos-positive cells (Fig. 5) concur with such morphological differences, showing a different density between wild-type and ha(33) fish. Despite having the same number of otoliths, the hypergravity-induced brain activities of ha(3-3) and wildtype fish were not equal at all and their brains responded differently to $3 \mathrm{G}$.

The brain activity differences between ha(3-3) and wild-type fish can be explained by the delay in the appearance of utriculus in ha(3-3) fish. Our previous study showed that in embryos and following the early juvenile stages, no ha fish have three pairs of otoliths, and it is at later stages that some ha fish form utricular otoliths. This implies that the critical stage for the development and establishment of gravity perception in medaka fish is in the early developmental stages and that having utricular otoliths that emerge in adulthood is too late for the establishment of gravity perception.

High numbers of c-Fos-positive cells in wild-type brains were always associated with low counts in both $h a(3-3)$ and $h a(2-2)$ brains in the TE, flm, and PON+CAN regions (Fig. 5). This is explained by the known functions of these brain regions. The PON+CAN contains the octaval nerve (nerve from vestibular organ: nerve VIII) nucleus, and fIm is connecting nerve VIII signal with eye movement information. The TE is, in general, related to behavior. Thus, the data for the TE, flm, and $\mathrm{PON}+\mathrm{CAN}$ in Fig. 5 indicate that information received from hypergravity exposure was transmitted normally to the appropriate brain regions in wild-type fish, whereas the vestibular apparatuses of both ha mutants could not sense hypergravity properly and the related signals were not transmitted to the brain nuclei.

Though not as marked, a similar tendency was seen in the TS and TO. The TO, which is also connected to the $T E$, is the brain region mostly closely associated with visual information (Northmore, 1981), while the TS deals with sensory information (auditory, lateral line, somatosensory). In addition, reciprocal connections with the TO exist (Northmore, 2011). This may explain why the cell density data of the TS and TO regions show a similar pattern (Fig. 5).

An interesting exception was the SPV region, which showed the highest density in ha(2-2) fish and the lowest in wild-types. The SPV is the deepest layer (the sixth layer) of the TO and it is composed of densely packed neuronal cell bodies, which are related to body movement, not visual information. Though the utricular otoliths in ha(3-3) do not perform their gravity-sensing function as expected, for a particular brain region like the SPV, the existence of the utricular otoliths (lapilli) results in different responses from ha(2-2) fish (which have no lapilli). At present, we have no explanation for the increased density of c-Fos-positive cells caused by hypergravity in this brain region of ha(2-2) fish, as well as the low values for $h a(3-3)$ and wild-type fish, and further studies are necessary.

The present results showed that the utricular otolith (lapillus) is the otolith mainly responsible for the gravity perception of medaka and that ha mutants can serve as a model for fish born and raised in microgravity. The ha(3-3) fish, though equipped as adults with a full set of otoliths, cannot develop gravity perception due to a lack of utricular otoliths at a young age, and may, in addition to ha(2-2), also be used as a model for microgravity-grown fish.

\section{Acknowledgments}

Helpful comments of Prof. Makoto Okuno on the centrifuge experiments are greatly appreciated.

\section{References}

Fuller, P.M., Jones, T.A., Jones, S.M. and Fuller, C.A. (2004) Evidence for macular gravity receptor modulation of hypothalamic, limbic and autonomic nuclei. Neuroscience, 129, 461-471.

Furukawa, R. and ljiri, K. (2002) Swimming behavior of larval medaka fish under microgravity. Adv. Space Res., 30, 733-738.

Gustave Dit Duflo, S., Gestreau, C., Lacour, M. (2000) Fos expression in the rat brain after exposure to gravito-inertial force changes. Brain Res., 861, 333344.

Hunt, S.P., Pini, A. and Evan, G. (1987) Induction of c-foslike protein in spinal cord neurons following sensory stimulation. Nature, 328, 632-634.

Ijiri, K. (1995a) Fish mating experiments in space - What 
it aimed at and how it was prepared. Biol. Sci. Space, $\mathbf{9}$, 3-16.

ljiri, K. (1995b) Medaka fish had the honor to perform the first successful vertebrate mating in space. Fish Biol. J. MEDAKA, 7, 1-10.

ljiri, K. (1998) Development of space-fertilized eggs and formation of primordial germ cells in the embryos of medaka fish. Adv. Space Res., 21, 1155-1158.

ljiri, K. (2000) Vestibular and visual contribution to fish behavior under microgravity. Adv. Space Res., 25, 1997-2006.

ljiri, K. (2003) Life-cycle experiments of medaka fish aboard the international space station. Adv. Space Biol. Med., 9, 201-216.

Ijiri, K., Mizuno, R. and Eguchi, H. (2003) Use of an otolith-deficient mutant in studies of fish behavior in microgravity. Adv. Space Res., 32, 1501-1512.

Ishikawa, Y. Medaka Histological Atlas. Edited by the Editorial Board of Medaka Histological Atlas of NBRP Medaka. http://rcshigen.lab.nig.ac.jp/mb_src/ medakabook2/index.php

Lai, C., Tse, Y., Shum, D.K.Y., Yung, K.K.L and Chan, Y. (2004) Fos expression in otolith-related brainstem neurons of postnatal rats following off-vertical axis rotation. J. Comp. Neurol., 470, 282-296.

Mayer, U. and Bischof, H.J. (2012) Brain activation pattern depends on the strategy chosen by zebra finches to solve an orientation task. J. Exp. Biol., 215, 426-434.

Mizuno, R. and Ijiri, K. (2003) Otolith formation in a mutant medaka with a deficiency in gravity-sensing. Adv. Space Res., 32, 1513-1520.

Noro, S., Yamamoto, N., Ihsikawa, Y., Ito, H. and ljiri, K. (2007) Studies on the morphology of the inner ear and semicircular canal endorgan projections of ha, a medaka behavior mutant. Fish Biol. J. MEDAKA, 11, 31-41.

Northmore, D.P. (1981) Visual localization after rearrangement of retinotectal maps in fish. Nature, 293, $142-144$.

Northmore, D.P. (2011) The Optic Tectum. In Encyclopedia of Fish Physiology : From Genome to Environment (Ed. A.P. Farrell) vol. 1, 131-142.

Okuyama, T., Suehiro, Y., Imada, H., Shimada, A., Naruse, K., Takeda, H., Kubo, T. and Takeuchi, H. (2011) Induction of c-fos trascription in the medaka brain (Oryzias latipes) in response to mating stimuli. Biochem. Biophys. Res. Commun., 404, 453-457.

Rasband, W.S., ImageJ, (1997-2013) U. S. National Institutes of Health, Bethesda, Maryland, USA, http:// imagej.nih.gov/ij/.

Shimomura-Umemura, S. and Ijiri, K. (2006) Effect of hypergravity on expression of the immediate early gene, c-fos, in central nervous system of medaka (Oryzias latipes). Adv. Space Res., 38, 1082-1088. 